\title{
Comparison of image-assisted versus traditional fundus examination
}

\section{Kristen Brown ${ }^{1,2}$ \\ Jeanette M Sewell ${ }^{1,2}$ \\ Clement Trempe ${ }^{2}$ \\ Tunde Peto ${ }^{3}$ \\ Thomas G Travison ${ }^{4,5}$}

'New England College of Optometry, ${ }^{2} \mathrm{New}$ England Eye Institute, Boston, MA, USA; ${ }^{3}$ NIHR Biomedical Research Centre for Ophthalmology, Moorfields Eye Hospital NHS Foundation Trust and UCL Institute of Ophthalmology, London, UK; ${ }^{4}$ Department of Medicine, ${ }^{5}$ Department of Biostatistics, Boston University School of Medicine, Boston, MA, USA
Correspondence: Kristen Brown New England Eye Institute Inc, 930 Commonwealth Avenue West, Suite 2, Boston, MA 02215, USA

$\mathrm{Tel}+\mathrm{I} 6172622020$

Fax +16172366323

Email brownk@neco.edu
This article was published in the following Dove Press journal:

Eye and Brain

13 February 2013

Number of times this article has been viewed

Background: The purpose of this study was to evaluate the ability of image-assisted fundus examination to detect retinal lesions compared with traditional fundus examination.

Methods: Subjects were imaged using a nonmydriatic ultrawide field scanning laser ophthalmoscope. After imaging, subjects underwent both a traditional and an image-assisted fundus examination, in random order. During the image-assisted method, ultrawide field scanning laser ophthalmoscopic images were reviewed in conjunction with a dilated fundus examination. Lesions detected by each method were assigned to one of three regions, ie, optic disc, posterior pole/macula, or mid-to-peripheral retina. Discrepancies between the image-assisted and the traditional examination methods were adjudicated by a retinal ophthalmologist.

Results: In total, 170 subjects (339 eyes) were recruited. Agreement between image-assisted and traditional fundus examination varied by lesion type and was excellent for staphyloma (kappa 0.76), fair for suspicious cupping (kappa 0.66), drusen in the posterior pole/macula and mid-to-peripheral retina $(0.45,0.41)$, retinal pigment epithelial changes in the posterior pole/macula (0.54), peripheral retinal degeneration (0.50), cobblestone $(0.69)$, vitreoretinal interface abnormalities (0.40), and vitreous lesions (0.53). Agreement was poor for hemorrhage in the mid-to-peripheral retina (kappa 0.33 ), and nevi in the mid-to-peripheral retina $(0.34)$. When the methods disagreed, the results indicated a statistically significant advantage for the image-assisted examination in detecting suspicious cupping $(P=0.04)$, drusen in the posterior pole/macula and mid-to-peripheral retina $(P=0.004, P<0.001)$, retinal pigment epithelial changes in the posterior pole/macula $(P=0.04)$, nevi in the posterior pole/macula and mid-toperipheral retina $(P=0.01, P=0.007)$, peripheral retinal degeneration $(P<0.001)$, hemorrhage in the mid-to-peripheral retina $(P=0.01)$, and vitreous lesions $(P<0.001)$.

Conclusion: Image-assisted fundus examination may enhance detection of retinal lesions compared with traditional fundus examination alone.

Keywords: imaging, ultrawide field, image-assisted fundus examination

\section{Introduction}

Timely detection and treatment of ocular disease can lower the risk of vision loss and reduce health care costs, particularly for those with diabetes and those at risk for agerelated macular degeneration and glaucoma. ${ }^{1-4}$ The use of nonmydriatic digital retinal imaging in the assessment and management of ocular disease is becoming increasingly widespread. Retinal imaging (fundus photography) has been used extensively in photodocumentation for clinical purposes, as well as in research, telemedicine, and patient education during routine eye care..$^{5-9}$

Previous studies have found that nonmydriatic digital retinal imaging alone exhibits performance comparable with dilated fundus examination and with dilated fundus 
photography for the detection of diabetic retinopathy. ${ }^{10-15}$ Some studies have demonstrated that fundus photography is superior to dilated fundus examination, particularly for the detection of subtle retinal lesions such as microaneurysms, small retinal hemorrhages, and neovascularization at the disc or elsewhere. ${ }^{10,16-21}$ Cavallerano et al showed that nonmydriatic imaging may be an acceptable alternative to an annual dilated fundus examination for determining the level of diabetic retinopathy. ${ }^{22}$ In the detection of ocular disease, apart from diabetic retinopathy, Chow et al demonstrated strong agreement between nonmydriatic digital retinal imaging and dilated ophthalmoscopy. ${ }^{23}$ Most digital instruments for fundus photography capture a 30 or 45 degree field of view. ${ }^{30}$ Recent advances in technology have allowed for development of imaging methods that enable the entire retina to be imaged. Up to 200 degrees of the retina are captured in one Optomap ${ }^{\circledR}$ ultrawide field scanning laser ophthalmoscope (UWF SLO) image. In addition, eye steering can be used to capture more peripheral views of the fundus, out to the ora in some cases. Recent studies have found nonmydriatic digital retinal imaging with UWF SLO to be a reliable screening tool for diabetic retinopathy and choroidal lesions. ${ }^{24-28}$ Silva et al found excellent agreement between nonmydriatic Optomap UWF SLO images, Early Treatment Diabetic Retinopathy Study (ETDRS) photographs, and dilated ophthalmoscopy. ${ }^{29}$

To our knowledge, this is the first study to evaluate the ability of nonmydriatic imaging to enhance a dilated fundus examination in the detection of retinal pathology, diabetic or otherwise. Therefore, the goal of this study was to evaluate the effectiveness of nonmydriatic digital retinal imaging to enhance detection of retinal lesions in conjunction with a dilated fundus examination.

\section{Materials and methods}

In this cross-sectional study, traditional fundus examination alone was compared with image-assisted fundus examination, utilizing nonmydriatic Optomap UWF SLO digital retinal images to serve as a guide for the dilated examination. A total of 170 subjects were recruited from the primary care and retina practices of the New England Eye Institute and from the greater Boston community. Approximately one-third of subjects, all patients of the New England Eye Institute, were invited by letter to participate based on a known history of ocular findings such as glaucoma, drusen, nevi, and or peripheral retinal degeneration. The remaining subjects were recruited through a community advertisement in order to include subjects with no known eye disease. Initial eligibility of these subjects was determined by telephone.
Subjects were included in the study only if they met all of the following inclusion criteria: age 18 years or older, willingness to undergo nonmydriatic retinal imaging and pharmacologic pupil dilation, and willingness to sign the institutionally approved informed consent specifically designed for this study. Subjects were excluded from this study if they had a history of adverse reaction to dilation or dilating drops, if they had an ocular condition that would preclude dilation, if they were using drops that would alter pupil size or affect pupil dilation, or if they had a history of epilepsy sensitive to single flash photography. The study design was consistent with the tenets of the Declaration of Helsinki, and all procedures were approved by the institutional review board of the New England College of Optometry.

Subjects were imaged with a scanning laser ophthalmoscope (Optomap P200C, Optos Plc, Dunfermline, Scotland). The $\mathrm{P} 200 \mathrm{C}$ is an ultrawide field digital imaging device capable of capturing an image of the retina out to the far periphery without scleral depression or contact with the cornea. The P200C uses red $(633 \mathrm{~nm})$ and green $(532 \mathrm{~nm})$ lasers to produce a digital image which is displayed on a PC monitor screen. The red and green low-powered laser beams scan in two dimensions over the retina. Light reflected from the retina is then detected and transformed into a digital computer image. Previously, scanning laser ophthalmoscope technology was constrained to imaging small fields of view. The P200C extends the range of scanning laser ophthalmoscope technologies by capturing ultrawide field, high resolution images without the need for pupil dilation.

The P200C system scans the two frequencies of laser light simultaneously. Light from the imaging lasers are combined into a single beam that scans quickly in the vertical axis and horizontal axis. The system can capture a single image of almost the entire retina in 0.4 seconds. The two wavelengths penetrate retinal structures to different degrees, each wavelength providing information to support interpretation and diagnosis. Images are displayed as a full color composite and can be reviewed instantly. Images can be enlarged, annotated, and/or separated into their color components. P200C resolution is 14 microns in the Optomap Plus mode and 17 microns in Optomap mode, with $3000 \times 3900(12 \mathrm{M})$ pixels per channel.

Images were taken by a trained and certified technician through an undilated pupil on all subjects. A total of six 200 degree images were taken per eye, ie, one centrally focused, one centrally focused with higher resolution, and four steered (superior, inferior, nasal, and temporal). The latter is known as eye-steering and this maximizes the chance 
of detecting lesions beyond the 200 degrees centered on the posterior pole, especially in the far inferior and far superior retina where lid artifact might otherwise be an issue.

After imaging, the subjects' eyes were dilated using $2.5 \%$ phenylephrine and $1 \%$ tropicamide ophthalmic solution. Subjects then underwent two separate methods of examination by qualified and experienced optometrists, ie, a traditional fundus examination and an image-assisted fundus examination. The two examinations were performed in one sitting, one immediately following the other, in random order. The examiners were randomized at the subject level to perform either the traditional or the image-assisted examination, but not both on the same subject. Each examiner was masked to the findings of the other examiner and to any previous findings in the clinical record. Examiners were not aware of which subjects had a history of ocular disease.

The traditional fundus examination consisted of slit-lamp biomicroscopy and binocular indirect ophthalmoscopy. Examiners followed a standard examination protocol utilizing a 20D condensing lens with binocular indirect ophthalmoscopy and a 90D condensing lens with slit-lamp biomicroscopy. The image-assisted method consisted of a dilated fundus examination as well as digital retinal image review. During image-assisted fundus examination, all UWF SLO retinal images (six per eye) for each subject were reviewed in advance and or in conjunction with the dilated fundus examination (slit-lamp biomicroscopy and binocular indirect ophthalmoscopy). Images were reviewed on a 17 inch monitor (resolution $1280 \times 1024$ at $75 \mathrm{~Hz}$ ). During the image-assisted method, examiners followed a standard image review protocol on each subject which included review of each image in the composite (red plus green laser) mode, the red laser only mode and the green laser only mode. Zoom, gamma, and contrast were adjusted as needed to highlight features (eg, to maximize visualization of the retinal nerve fiber layer). Cup to disc ratio was quantified for each eye independently by utilizing the "cup to disc ratio" image review tool, and lesion size was measured in pixels utilizing the "measure distance" image review tool.

Lesions detected by each examiner were recorded on separate data collection forms. Examiners documented each of 16 lesion types individually as either "definite" (present) or "no evidence" (absent) for each eye separately. Specific criteria were utilized to assist in the designation of the presence or absence of a lesion. For example, in order for lesions to be considered definitely present (eg, retinal pigment epithelial changes, scarring), the lesion must have measured 25 pixels or more in length or width, with the exception of drusen and retinal hemorrhages. For these lesions, the observation of one druse or one dot/blot hemorrhage would be sufficient to result in a mark indicating the definite presence of the lesion. Suspicious cupping was defined as $0.50 \mathrm{C} / \mathrm{D}$ or greater, asymmetry of 0.20 or more between the eyes, and or the presence of notching. In denoting the location of lesions, examiners utilized a grid delineating nine areas of the retina (Figure 1). If a lesion was present, examiners also noted the area in which the lesion was found. Lesions found in one or more areas were designated as such. Lesions detected were then grouped into one of three larger regions, ie, the optic disc, corresponding to lesions involving the optic disc only; the posterior pole/macula, defined as the area inside the vascular arcades and within one disc diameter nasal to the optic disc (corresponding to area 1); or the mid-to-peripheral retina, defined as the area between the vascular arcades and the ora serrata (corresponding to areas 2-9). Lesions detected by traditional fundus examination included those seen by slit lamp biomicroscopy and or binocular indirect ophthalmoscopy. Lesions detected by image-assisted fundus examination included those seen by slit-lamp biomicroscopy, binocular indirect ophthalmoscopy, and/or as seen in the UWF SLO images.

When the traditional and image-assisted methods disagreed on any component of the examination, a retinal ophthalmologist performed an adjudication to determine the presence or absence of a lesion and thus served as the reference standard. Adjudication (for one or more lesions in either eye) was performed on 70 subjects. Sixty-six of these subjects were adjudicated the same day the traditional and image-assisted examinations were performed. Four subjects returned for adjudication within one month of the traditional

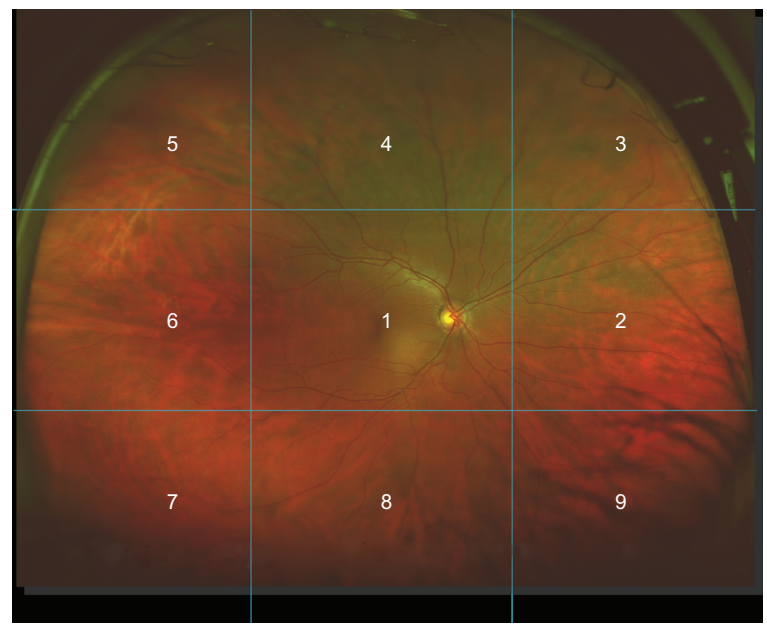

Figure I Ultrawide field scanning laser ophthalmoscope (Optomap $\left.{ }^{\circledR}\right)$ image. Note: Areas I-9 are delineated to denote location of lesions during image review (Copyright Optos, All rights reserved, reprinted with permission). 
and image-assisted examinations. The retinal specialist was masked to both the examiner and to the method that indicated the presence or absence of a lesion. The retinal specialist determined presence of absence of lesions in question utilizing slit-lamp biomicroscopy, binocular indirect ophthalmoscopy, scleral indentation, and or review of UWF SLO images. When the two methods agreed they were assumed to be correct, and no adjudication was performed.

Exact agreement between the traditional and imageassisted methods was assessed. Each lesion $\times$ area combination was analyzed separately. Agreement between methods was dependent both upon each method noting the lesion as present (or absent) as well as by each method identifying the correct location of the lesion, within two clock hours. When the methods differed in their assessment, adjudication was employed. The degree of adjudicators' agreement with each method was then computed. The difference between rate of confirmation for the methods by the adjudication process was then assessed by comparing these proportions, combining observations in OD and OS eyes. In order to acknowledge the fact that multiple observations (eg, OD and OS) may have arisen from individual subjects in this analysis, the statistical significance of the difference in these proportions was determined using the generalized estimating equations method, which allows for repeated observations on individual subjects. Analyses were considered statistically significant if null hypotheses could be rejected at the 0.05 level. Kappa values were calculated from the generalized estimating equations.

\section{Results}

The sample had a mean ( \pm standard deviation) age of $48 \pm 17$ years and was predominantly white $(65 \%)$ and female $(58 \%)$. Twenty-one percent were Black, 8\% Asian, 3\% Hispanic, and $2 \%$ other. Medical history was assessed according to selfreport, with $6 \%$ having diabetes, $19 \%$ having hypertension, and 14\% having elevated cholesterol. Fifty-seven (19\%) of 300 patients from the New England Eye Institute responded to the letter inviting them to participate in the study. Of those who responded to an advertisement in a community paper, 115 were eligible to participate. Failure to keep scheduled appointments did not differ significantly from subjects who participated by age, gender, ethnicity or race, and medical or ocular history.

Table 1 demonstrates the prevalence or overall proportion of eyes in which a lesion was identified by either both methods of examination or by adjudication. Prevalence of lesions ranged from approximately $0.88 \%$ (3/339) observations of scar in the posterior pole/macula to $45.1 \%$ (153/339) observations of peripheral retinal degeneration.

Table 2 outlines the exact agreement between the image-assisted and traditional examination methods. For lesions that were prevalent (5\% or more), agreement was very good. Agreement was excellent for optic disc staphyloma (kappa 0.76) and poor for hemorrhage in the mid-to-peripheral retina $(0.33)$ and choroidal nevus in the mid-to-peripheral retina (0.34).

Table 3 reports the number of lesions present and the proportion of these lesions detected by each method of examination (as indicated either by agreement between the two examination methods or by adjudication). For example, the image-assisted method detected $90.6 \%$ (58/64) of drusen in the posterior pole/macula compared with $43.8 \%(28 / 64)$ of posterior pole/macula drusen detected by the traditional fundus examination alone. Nearly half of the lesions detected in the posterior pole/macula region were drusen (64/131). Only 15 of the 419 lesions found in the mid-to-peripheral retina were retinal holes or tears in this study (4.4\% of all eyes), which was not enough to determine a significant difference between the methods to detect such lesions. The majority $(122 / 128)$ of vitreoretinal interface abnormalities detected in this study were white without pressure which was detected

Table I Prevalence of lesions $(n=170,339$ eyes)*

\begin{tabular}{ll}
\hline Region and lesion & Prevalence \\
\hline Optic disc & \\
Suspicious cupping ${ }^{\ddagger}$ & $27.1 \%(92)$ \\
Staphyloma & $5.9 \%(20)$ \\
Posterior pole/macula & \\
Drusen & $18.9 \%(64)$ \\
RPE changes & \\
Hemorrhage & $11.8 \%(40)$ \\
Scar & $2.4 \%(8)$ \\
Choroidal nevus & $0.88 \%(3)$ \\
Mid-to-peripheral retina & $4.72 \%(16)$ \\
Drusen & \\
Peripheral retinal degeneration ${ }^{\|}$ & $14.5 \%(49)$ \\
Hemorrhage & $45.1 \%(153)$ \\
Scar & $5.9 \%(20)$ \\
Choroidal nevus & $3.8 \%(13)$ \\
Cobblestone & $5.9 \%(20)$ \\
Retinal hole or tear & $6.19 \%(2 I)$ \\
Vitreoretinal interface abnormality & $4.4 \%(15)$ \\
Vitreous & $37.8 \%(I 28)$ \\
\hline
\end{tabular}

Notes: *One subject missing OS data; ${ }^{\ddagger}$ defined as 0.50 or greater cup to disc ratio, 0.20 or greater asymmetry between $\mathrm{OD}$ and $\mathrm{OS}$, and or notching; 'setinal pigment epithelial (RPE) changes including RPE hypertrophy, RPE hyperplasia and RPE hypoplasia; "lincluding RPE hypertrophy, RPE hyperplasia, RPE hypoplasia, lattice, snail tracking, cystoid degeneration, and other peripheral degenerations (pigmented or nonpigmented of size 25 pixels or greater); * numerators indicate the number of observations of lesions present as determined by both methods or in the case of disagreement, by adjudication. 
Table 2 Exact agreement between traditional and image-assisted ophthalmoscopy

\begin{tabular}{lll}
\hline Region and lesion & $\begin{array}{l}\text { Exact agreement } \\
\text { OD/OS }\end{array}$ & $\begin{array}{l}\text { Kappa } \\
\text { OD/OS }\end{array}$ \\
\hline Optic disc region & & \\
Suspicious cupping & $87 \% / 88 \%$ & $0.64 / 0.68$ \\
$\quad$ Staphyloma & $98 \% / 96 \%$ & $0.79 / 0.73$ \\
Posterior pole/macula & & \\
Drusen & $85 \% / 86 \%$ & $0.42 / 0.48$ \\
RPE change & $92 \% / 87 \%$ & $0.63 / 0.44$ \\
Hemorrhage & $98 \% / 98 \%$ & $0.66 / 0.76$ \\
Scar & $99 \% / 99 \%$ & $0.80 / 0.86$ \\
Choroidal nevus & $95 \% / 95 \%$ & $0.30 / 0.33$ \\
Mid-to-peripheral retina & & \\
Drusen & $88 \% / 89 \%$ & $0.37 / 0.44$ \\
Peripheral retinal degeneration & $74 \% / 77 \%$ & $0.45 / 0.55$ \\
Hemorrhage & $95 \% / 94 \%$ & $0.32 / 0.34$ \\
Scar & $99 \% / 96 \%$ & $0.85 / 0.61$ \\
Choroidal nevus & $94 \% / 93 \%$ & $0.26 / 0.42$ \\
Cobblestone & $96 \% / 96 \%$ & $0.65 / 0.72$ \\
Retinal hole or tear & $97 \% / 96 \%$ & $0.60 / 0.52$ \\
Vitreoretinal interface abnormality & $73 \% / 73 \%$ & $0.41 / 0.39$ \\
Vitreous & $82 \% / 82 \%$ & $0.53 / 0.53$ \\
\hline
\end{tabular}

Note: $P<0.001$ for all lesions OD/OS.

in $36 \%$ of all eyes. The image-assisted method detected $118 / 128(92.2 \%)$ of all vitreoretinal interface abnormalities while the traditional examination detected 70/128 (54.7\%). When the methods disagreed for any lesion type, the imageassisted method was correct in $75 \%$ of the disagreements (69/92, $P=0.001)$.

Table 4 examines cases where the image-assisted and traditional methods disagreed for each lesion by region combination. When the image-assisted and traditional

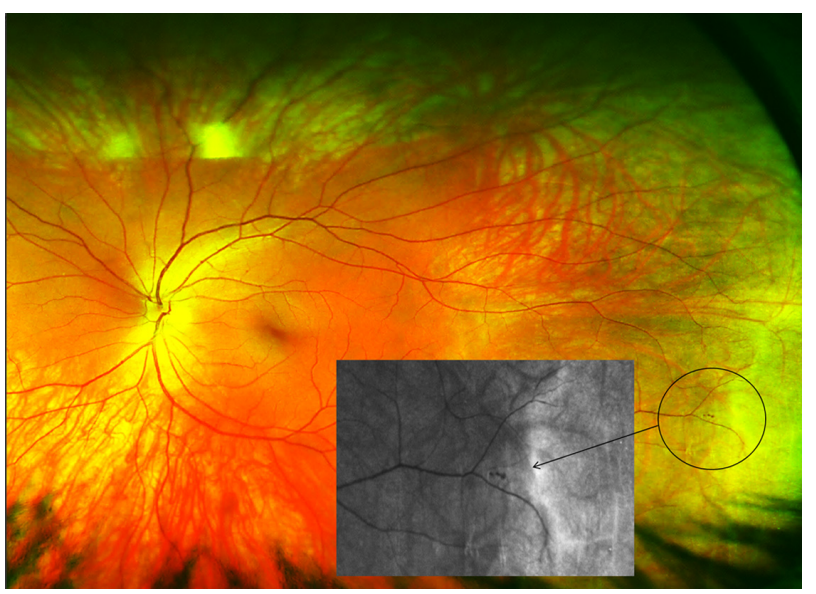

Image I Nonmydriatic, ultrawide field image of a peripheral retinal hemorrhage adjacent to an area of retinal thinning (area in circle) in a 26-year-old, healthy, asymptomatic subject.

Notes: The insert is a green laser channel view of the same peripheral hemorrhage. Small yellow artifact at center of image and eyelashes at lower edge of image. Brightness and contrast of image have been enhanced to increase visibility of lesion. methods disagreed on the presence or absence of a particular lesion type, the analysis indicates a statistically significant advantage for the image-assisted fundus examination. For instance, where the methods disagreed on the presence or absence of drusen, adjudication sided in favor of the imageassisted method in 35 of 49 cases in the posterior pole/macula region $(71 \%, P<0.004)$ and in 33 of 39 cases in the midto-peripheral retina $(85 \%, P<0.001)$. When the methods disagreed regarding the presence or absence of the lesion "peripheral retinal hemorrhage", adjudication sided in favor of the image-assisted method in 16 of the 19 cases $(84 \%$, $P<0.01)$. Stratified analyses indicate consistent results between examiners. The image-assisted method was preferred by the adjudicator in the case of both examiners $(76 \%$ of the time for examiner A and 74\% for examiner B).

\section{Discussion}

This cross-sectional study found good agreement between image-assisted and traditional fundus examination. In cases of disagreement, the adjudicator agreed with the imageassisted method in over $70 \%$ of cases. This suggests that adding nonmydriatic imaging, Optomap UWF SLO in this case, to the clinical examination can improve the examiner's ability to detect or rule out lesions.

Our findings are similar to those of previous studies that compared traditional dilated ophthalmoscopy with fundus photography. The sensitivity of dilated ophthalmoscopy in previous studies ranges from $32 \%$ to $82 \%$ for the detection of diabetic retinopathy. ${ }^{5,16-18,21}$ The strength of this study is that it includes peripheral retinal lesions as well as macular and optic disc changes. There was a higher rate of detection of posterior pole/macula region lesions using the imageassisted method in this study (90.1\%). This illustrates the potential advantage of utilizing fundus imaging to enhance the detection of subtle retinal lesions compared with ophthalmoscopy alone.$^{5,16-18,21}$ Similar conclusions were reached by other studies regarding the detection of subtle retinal lesions by fundus photography, ${ }^{16,19,20,32-34}$ and it was hypothesized that retinal photography has greater sensitivity in detecting subtle abnormalities than clinical examination alone. ${ }^{20}$ This was reinforced in the Beaver Dam Eye study, where more subjects were found to have retinal hemorrhages on imaging than on examination only (Image 1). ${ }^{19}$

In previous studies assessing ultrawide field imaging, researchers have found good agreement between nonmydriatic UWF SLO image review alone and dilated clinical examination for the detection of diabetic retinopathy. Silva et al compared nonmydriatic Optomap UWF SLO image 
Table 3 Proportion of observations agreeing with adjudication $(n=170,339$ eyes)*

\begin{tabular}{|c|c|c|c|c|}
\hline \multirow[t]{2}{*}{ Region and lesion } & \multicolumn{2}{|l|}{ Image-assisted } & \multicolumn{2}{|l|}{$\underline{\text { Traditional }}$} \\
\hline & Lesion present & Lesion absent & Lesion present & Lesion absent \\
\hline \multicolumn{5}{|l|}{ Optic disc } \\
\hline Suspicious cupping ${ }^{\ddagger}$ & $88 \%(8 \mathrm{I} / 92)$ & $98.4 \%(243 / 247)$ & $76.1 \%(70 / 92)$ & $97.2 \%(240 / 247)$ \\
\hline Staphyloma & $100 \%(20 / 20)$ & $99.7 \%(3 \mid 8 / 319)$ & $70 \%(14 / 20)$ & $99.1 \%(316 / 319)$ \\
\hline Region total & $90.2 \%(101 / 1 / 2)$ & $99.1 \%(561 / 566)$ & $75 \%(84 / 1 / 2)$ & $98.2 \%(556 / 566)$ \\
\hline \multicolumn{5}{|l|}{ Posterior pole/macula } \\
\hline Drusen & $90.6 \%(58 / 64)$ & $96.7 \%(266 / 275)$ & $43.8 \%(28 / 64)$ & $100 \%(275 / 275)$ \\
\hline RPE changes ${ }^{\S}$ & $82.5 \%(33 / 40)$ & $98 \%(293 / 299)$ & $70 \%(28 / 40)$ & $95.3 \%(285 / 299)$ \\
\hline Hemorrhage & $100 \%(8 / 8)$ & $99.4 \%(329 / 331)$ & $62.5 \%(5 / 8)$ & $99.7 \%(330 / 331)$ \\
\hline Scar & $100 \%(3 / 3)$ & $100 \%(336 / 336)$ & $66.7 \%(2 / 3)$ & $99.7 \%(335 / 336)$ \\
\hline Choroidal nevus & $100 \%(16 / 16)$ & $99.4 \%(321 / 323)$ & $6.25 \%(1 / 16)$ & $100 \%(323 / 323)$ \\
\hline Region total & $90.1 \%(|| 8 /|3|)$ & $98.8 \%(1545 / 1564)$ & $48.9 \%(64 /|3|)$ & $99 \%(1548 / 1564)$ \\
\hline \multicolumn{5}{|l|}{ Mid-to-peripheral retina } \\
\hline Drusen & $91.8 \%$ (45/49) & $98.3 \%(285 / 290)$ & $28.6 \%(14 / 49)$ & $99.7 \%(289 / 290)$ \\
\hline Peripheral retinal degeneration" & $88.9 \%(136 / 153)$ & $94.6 \%(176 / 186)$ & $73.9 \%(113 / 153)$ & $89.2 \%(166 / 186)$ \\
\hline Hemorrhage & $90 \%(18 / 20)$ & $99.4 \%(3|7 / 3| 9)$ & $15 \%(3 / 20)$ & $100 \%(3|9 / 3| 9)$ \\
\hline Scar & $92.3 \%(12 / 13)$ & $99.7 \%(325 / 326)$ & $69.2 \%(9 / 13)$ & $99.4 \%(324 / 326)$ \\
\hline Choroidal nevus & $90 \%(18 / 20)$ & $99.4 \%(317 / 319)$ & $30 \%(6 / 20)$ & $98.4 \%(3 / 4 / 319)$ \\
\hline Cobblestone & $100 \%(2|/ 2|)$ & $99.7 \%(3|7 / 3| 8)$ & $66.7 \%(\mid 4 / 2 I)$ & $98.1 \%(3 / 2 / 318)$ \\
\hline Retinal hole or tear & $73.3 \%(11 / 15)$ & $99.4 \%(322 / 324)$ & $60 \%(9 / 15)$ & $99.4 \%(322 / 324)$ \\
\hline Vitreoretinal interface abnormality & $92.2 \%(118 / 128)$ & $93.8 \%(198 / 2 I I)$ & $54.7 \%(70 / I 28)$ & $94.8 \%(200 / 211)$ \\
\hline Region total & $90.5 \%(379 / 419)$ & $98.4 \%(2257 / 2293)$ & $56.8 \%(238 / 419)$ & $98 \%(2246 / 2293)$ \\
\hline Vitreous & $93.8 \%$ (9l/97) & $97.1 \%$ (235/242) & $56.7 \%(55 / 97)$ & $96.7 \%(234 / 242)$ \\
\hline
\end{tabular}

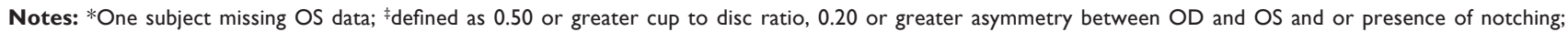
$\S$ including retinal pigment epithelial (RPE) hypertrophy, RPE hyperplasia, and RPE hypoplasia; "lincludes RPE hypertrophy, RPE hyperplasia, RPE hypoplasia, lattice, snail tracking, cystoid degeneration, and other peripheral degenerations (pigmented or nonpigmented of size 25 pixels or greater); *numerators indicate the number of observations of lesion presence by the relevant method and denominators indicate the number of observations according to either/both methods, or in the case of disagreement, adjudication.

Table 4 Evaluation of discordant results

\begin{tabular}{|c|c|c|c|}
\hline Region and lesion & $\begin{array}{l}\text { Image-assisted } \\
\text { correct* }\end{array}$ & $\mathbf{N}$ & $P$ value $\neq$ \\
\hline \multicolumn{4}{|l|}{ Optic disc } \\
\hline Suspicious cupping & 28 & 42 & 0.04 \\
\hline Staphyloma & 9 & 10 & 0.06 \\
\hline \multicolumn{4}{|l|}{ Posterior pole/macula } \\
\hline Drusen & 35 & 49 & 0.004 \\
\hline RPE changes & 25 & 37 & 0.04 \\
\hline Hemorrhage & 4 & 6 & 0.49 \\
\hline Scar & 2 & 2 & - \\
\hline Choroidal nevus & 15 & 17 & 0.01 \\
\hline \multicolumn{4}{|l|}{ Mid-to-peripheral retina } \\
\hline Drusen & 33 & 39 & $<0.001$ \\
\hline $\begin{array}{l}\text { Peripheral retinal } \\
\text { degeneration }\end{array}$ & 57 & 81 & $<0.001$ \\
\hline Hemorrhage & 16 & 19 & 0.01 \\
\hline Scar & 6 & 8 & 0.229 \\
\hline Choroidal nevus & 19 & 23 & 0.007 \\
\hline Cobblestone & 13 & 14 & 0.02 \\
\hline Retinal hole or tear & 7 & 12 & 0.59 \\
\hline $\begin{array}{l}\text { Vitreoretinal interface } \\
\text { abnormality }\end{array}$ & 69 & 92 & $<0.001$ \\
\hline Vitreous & 47 & 57 & $<0.001$ \\
\hline
\end{tabular}

Notes: *Image-assisted results correct and traditional method incorrect, per adjudication; "test of proportions via generalized estimating equations. review with dilated ETDRS standard seven-field $35 \mathrm{~mm}$ photography and dilated clinical examination for evaluation of diabetic retinopathy. Exact agreement between UWF SLO image review alone and ETDRS photographs was $84 \%$ $[\mathrm{K}(\mathrm{W})=0.85]$ while agreement between UWF SLO image review and clinical examination was $70 \%[\mathrm{~K}(\mathrm{~W})=0.71]$. The authors also found that nonmydriatic UWF SLO image acquisition time was less than half the time taken to obtain dilated ETDRS photographs. ${ }^{29}$ Neubauer et al found good agreement between Optomap image review alone and clinical examination by three experienced retinal specialists, with unweighted kappas of $0.58,0.58$, and 0.51 in the assessment of diabetic retinopathy and macular edema. ${ }^{25}$

A limitation of this study is that two examiners were used to conduct the traditional and image-assisted fundus examinations, albeit in random order, and that not all examinations were adjudicated. The retinal specialist adjudicated only when the traditional and image-assisted methods disagreed and, therefore, did not serve as a completely independent gold standard. True sensitivity and specificity of the methods could not be determined..$^{32,33}$ Eye steering during imaging 
was included to maximize the potential of capturing lesions as peripheral in the retina as possible. This is not representative of the "one click" or single nonmydriatic image capture method employed in many ophthalmic clinics for routine screening of the retina. The goal of this study was to determine how UWF SLO imaging might maximize or enhance retinal lesion detection, not compare how single nonmydriatic imaging alone might compare with traditional fundus examination. Inclusion of additional subjects with lesions such as dry and wet age-related macular degeneration, as well as vascular and optic nerve pathologies, would improve the value of this research.

As the use of nonmydriatic imaging for the detection and management of ocular disease becomes more widespread, it is important to determine how such technology might be best incorporated into the clinical examination. It would be useful, for example, to determine the value of UWF SLO as a standalone screening tool by comparing UWF SLO review alone with traditional fundus examination. In addition, it would be valuable to determine if more retinal lesions might be detected when eye steering is included in the image review process compared with single-field nonmydriatic UWF SLO imaging, which may reflect current trends in retinal screening.

\section{Acknowledgments}

The authors would like to thank all those who contributed to the work presented in this paper, including Julie De Vita, William Sleight, and Sarah Dougherty Wood, who served as study investigators. Rebecca Gass served as patient care coordinator. Thanks are also extended to Jerry Cavallerano for his review of the manuscript. The National Institute for Health Research Biomedical Research Centre in Ophthalmology provided funding for Tunde Peto.

\section{Disclosure}

Optos Plc Scotland provided research funding for this study. None of the authors have any financial interest in Optos Plc. Part of this manuscript was presented as a poster at the Association for Research in Vision and Ophthalmology Annual Meeting, Fort Lauderdale, FL, May 3, 2010.

\section{References}

1. Javitt JC, Aiello LP, Bassi LJ, Chiang YP, Canner JK. Detecting and treating retinopathy in patients with type I diabetes mellitus. Savings associated with improved implementation of current guidelines. American Academy of Ophthalmology. Ophthalmology. 1991;98:1565-1573.

2. [No authors listed]. Early photocoagulation for diabetic retinopathy. ETDRS report number 9. Early Treatment Diabetic Retinopathy Study Research Group. Ophthalmology. 1991;98(Suppl 5):766-785.
3. [No authors listed]. A randomized, placebo-controlled, clinical trial of high-dose supplementation with vitamins $\mathrm{C}$ and $\mathrm{E}$, beta carotene, and zinc for age-related macular degeneration and vision loss: AREDS report no 8. Arch Ophthalmol. 2001;119:1417-1436.

4. Fong DS, Aiello L, Gardner TW, et al. Retinopathy in diabetes. Diabetes Care. 2004;27 Suppl 1:S84-S87.

5. Klein BE, Davis MD, Segal P, et al. Diabetic retinopathy. Assessment of severity and progression. Ophthalmology. 1984;91:10-17.

6. Klein R, Klein BE, Moss SE, Davis MD, DeMets DL. The Wisconsin epidemiologic study of diabetic retinopathy. II. Prevalence and risk of diabetic retinopathy when age at diagnosis is less than 30 years. Arch Ophthalmol. 1984;102:520-526.

7. Klein R, Klein BE, Linton KL. Prevalence of age-related maculopathy. The Beaver Dam Eye Study. Ophthalmology. 1992;99:933-943.

8. Constable IJ, Yogesan K, Eikelboom R, Barry C, Cuypers M. Fred Hollows lecture: digital screening for eye disease. Clin Experiment Ophthalmol. 2000;28:129-132.

9. [No authors listed]. Photocoagulation treatment of proliferative diabetic retinopathy: the second report of diabetic retinopathy study findings. Ophthalmology. 1978;85:82-106.

10. Lin DY, Blumenkranz MS, Brothers RJ, Grosvenor DM. The sensitivity and specificity of single-field nonmydriatic monochromatic digital fundus photography with remote image interpretation for diabetic retinopathy screening: a comparison with ophthalmoscopy and standardized mydriatic color photography. Am J Ophthalmol. 2002;134: 204-213.

11. Bursell SE, Cavallerano JD, Cavallerano AA, et al. Stereo nonmydriatic digital-video color retinal imaging compared with Early Treatment Diabetic Retinopathy Study seven standard field 35-mm stereo color photos for determining level of diabetic retinopathy. Ophthalmology. 2001;108:572-585.

12. Lopez-Bastida J, Cabrera-Lopez F, Serrano-Aguilar P. Sensitivity and specificity of digital retinal imaging for screening diabetic retinopathy. Diabet Med. 2007;24:403-407.

13. Ahmed J, Ward TP, Bursell SE, et al. The sensitivity and specificity of nonmydriatic digital stereoscopic retinal imaging in detecting diabetic retinopathy. Diabetes Care. 2006;29:2205-2209.

14. Boucher MC, Gresset JA, Angioi K, Olivier S. Effectiveness and safety of screening for diabetic retinopathy with two nonmydriatic digital images compared with the seven standard stereoscopic photographic fields. Can J Ophthalmol. 2003;38:557-568.

15. Lin DY, Blumenkranz MS, Brothers R. The role of digital fundus photography in diabetic retinopathy screening. Digital Diabetic Screening Group (DDSG). Diabetes Technol Ther. 1999;1:477-487.

16. Pugh JA, Jacobson JM, Van Heuven WA, et al. Screening for diabetic retinopathy. The wide-angle retinal camera. Diabetes Care. 1993;16: 889-895.

17. Kinyoun JL, Martin DC, Fujimoto WY, Leonetti DL. Ophthalmoscopy versus fundus photographs for detecting and grading diabetic retinopathy. Invest Ophthalmol Vis Sci. 1992;33:1888-1893.

18. Moss SE, Klein R, Kessler SD, Richie KA. Comparison between ophthalmoscopy and fundus photography in determining severity of diabetic retinopathy. Ophthalmology. 1985;92:62-67.

19. Klein R. Retinopathy in a population-based study. Trans Am Ophthalmol Soc. 1992;90:561-594.

20. Scott IU, Bressler NM, Bressler SB, et al. Agreement between clinician and reading center gradings of diabetic retinopathy severity level at baseline in a phase 2 study of intravitreal bevacizumab for diabetic macular edema. Retina. 2008;28:36-40.

21. Palmberg P, Smith M, Waltman S, et al. The natural history of retinopathy in insulin dependent juvenile-onset diabetes. Ophthalmology. 1981;88:613-618.

22. Cavallerano JD, Aiello LP, Cavallerano AA, et al. Nonmydriatic digital imaging alternative form of annual retinal examination in persons with previously documented no or mild diabetic retinopathy. Am J Ophthalmol. 2005;140:667-673. 
23. Chow SP, Aiello LM, Cavallerano JD, et al. Comparison of nonmydriatic digital retinal imaging versus dilated ophthalmic examination for nondiabetic eye disease in persons with diabetes. Ophthalmology. 2006;113:833-840.

24. Friberg TR, Pandya A, Eller AW. Non-mydriatic panoramic fundus imaging using a noncontact scanning laser-based system. Ophthalmic Surg Lasers Imaging. 2003;34:488-497.

25. Neubauer AS, Kernt M, Haritoglou C, et al. Nonmydriatic screening for diabetic retinopathy by ultra-widefield scanning laser ophthalmoscopy (Optomap). Graefes Arch Clin Exp Ophthalmol. 2008;246: 229-235.

26. Jain A, Shah SP, Tsui I, McCannel TA. The value of Optos Panoramic 200MA imaging for the monitoring of large suspicious choroidal lesions. Semin Ophthalmol. 2009;24:43-44.

27. Cheng SCK, Yap MKH, Goldschmidt E, et al. Use of the Optomap with lid retraction and its sensitivity and specificity. Clin Exp Optom. 2008;91:373-378.

28. Wilson PJ, Ellis JD, Macewen CJ, et al. Screening for diabetic retinopathy: a comparative trial of photography and scanning laser ophthalmoscopy. Ophthalmologica. 2010;224:251-257.
29. Silva PS, Cavallerano JD, Sun JK, et al. Nonmydriatic ultrawide field retinal imaging compared with dilated standard 7-field $35-\mathrm{mm}$ photography and retinal specialist examination for evaluation of diabetic retinopathy. Am J Ophthalmol. 2012;154:549-559.

30. Williams GA, Scott IU, Haller JA, et al. Single-field fundus photography for diabetic retinopathy screening: a report by the American Academy of Ophthalmology. Ophthalmology. 2004;111:1055-1062.

31. [No authors listed]. Photocoagulation for diabetic macular edema. Early Treatment Diabetic Retinopathy Study report number 1. Early Treatment Diabetic Retinopathy Study research group. Arch Ophthalmol. 1985;103:1796-1806.

32. Hutchinson A, McIntosh A, Peters J, et al. Effectiveness of screening and monitoring tests for diabetic retinopathy - a systematic review. Diabet Med. 2000;17:495-506.

33. Superstein R, Gomolin JE, Hammouda W, et al. Prevalence of ocular hemorrhage in patients receiving warfarin therapy. Can J Ophthalmol. 2000;35:385-389.

34. Taylor R, Lovelock L, Tunbridge WM, et al. Comparison of nonmydriatic retinal photography with ophthalmoscopy in 2159 patients: mobile retinal camera study. BMJ. 1990;301:1243-1247.
Eye and Brain

\section{Publish your work in this journal}

Eye and Brain is an international, peer-reviewed, open access journal focusing on clinical and experimental research in the field of neuro-ophthalmology. All aspects of patient care are addressed within the journal as well as basic research. Papers covering original research, basic science, clinical and epidemiological studies, reviews and

\section{Dovepress}

evaluations, guidelines, expert opinion and commentary, case reports and extended reports are welcome. The manuscript management system is completely online and includes a very quick and fair peer-review system, which is all easy to use. Visit http://www.dovepress.com/ testimonials.php to read real quotes from published authors. 